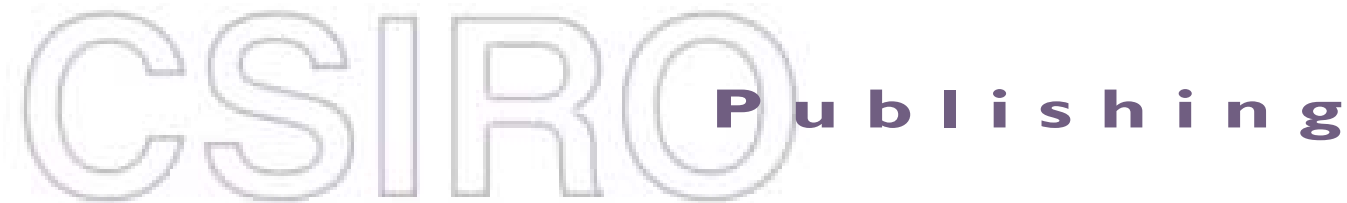

\section{Publications of the Astronomical Society of Australia}

Volume 19, 2002

(C) Astronomical Society of Australia 2002

An international journal of astronomy and astrophysics

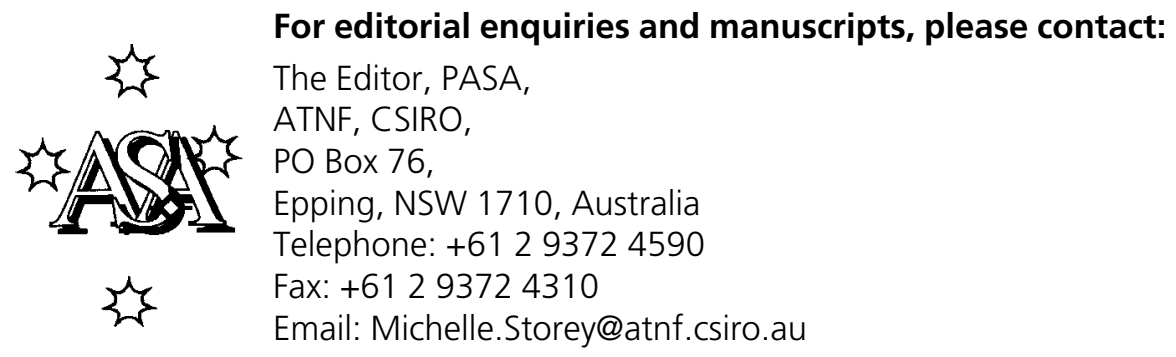

For general enquiries and subscriptions, please contact: CSIRO Publishing PO Box 1139 (150 Oxford St)

Collingwood, Vic. 3066, Australia

Telephone: +6139662 7666

Fax: +61 396627555

Email: publishing.pasa@csiro.au

C S I RO

PUBLISHING Published by CSIRO Publishing

for the Astronomical Society of Australia

www.publish.csiro.au/journals/pasa 


\title{
AAT/WFI Observations of the Extragalactic HI Cloud HIPASS J1712-64
}

\author{
Geraint F. Lewis ${ }^{1}$, Michael J. Irwin ${ }^{2}$, Rodrigo A. Ibata ${ }^{3}$ and Brad K. Gibson ${ }^{4}$ \\ ${ }^{1}$ Anglo-Australian Observatory, PO Box 296, Epping, NSW 1710, Australia \\ gfl@aaoepp.aao.gov.au \\ ${ }^{2}$ Institute of Astronomy, Madingley Rd, Cambridge, CB3 OHA, UK \\ mike@ ast.cam.ac.uk \\ ${ }^{3}$ Observatoire de Strabourg, 11, rue de l'Universite, F-67000, Strasbourg, France \\ ibata@pleiades.u-strasbg.fr \\ ${ }^{4}$ Centre for Astrophysics \& Supercomputing, Swinburne University, \\ PO Box 218, Hawthorn, Victoria 3122, Australia \\ bgibson@astro.swin.edu.au \\ Received 2002 February 11, accepted 2002 May 3
}

\begin{abstract}
AAT/WFI optical images of a candidate extragalactic HI cloud, HIPASS J1712-64, are presented. The $g$ and $r$ band CCD mosaic camera frames were processed using a new data pipeline recently installed at the AAO. The resultant stacked images reach significantly deeper levels than those of previous published optical imaging of this candidate, providing a detection limit $M_{g} \sim-7$ at a distance of $3 \mathrm{Mpc}$, the inferred distance to HIPASS J1712-64. However, detailed analysis of the images fails to uncover any stellar population associated with the HI emission. If this system is a member of the Local Group then it is pathologically different to other members. Hence, our observations reinforce earlier suggestions that this $\mathrm{HI}$ cloud is most likely Galactic in origin and not a Local Volume dwarf galaxy.
\end{abstract}

Keywords: galaxies: dwarf — Local Group — techniques: image processing

\section{Introduction}

All-sky HI surveys have found significant numbers of high velocity clouds (HVCs). Although various scenarios have been invoked to explain the disparate properties of the ensemble of HVCs, one population in particular, the isolated compact HVCs, remains a possible candidate for the continuing rain of material accreting onto the Milky Way and other Local Group galaxies (e.g. Braun \& Burton 1999; Blitz et al. 1999; Sembach et al. 2002), as predicted by models of hierarchical structure formation (e.g. Klypin et al. 1999).

The goal of the current market leader, the HI Parkes All-Sky Survey (HIPASS), is to map out the distribution of $\mathrm{HI}$ gas in the local universe, providing an accurate map of the HVC distribution at finer resolution and to better sensitivity levels than obtained previously.

Recently, Kilborn et al. (2000) announced the detection of an isolated compact HI cloud, HIPASS J171264 , with a systemic velocity of $\sim 450 \mathrm{~km} \mathrm{~s}^{-1}$. Transforming to the reference frame of the Local Group, J1712-64's velocity is $\sim 240 \mathrm{~km} \mathrm{~s}^{-1}$ which, assuming the cloud is extragalactic, places it at a distance of $\sim 3 \mathrm{Mpc}$ $\left(H_{0}=75 \mathrm{~km} \mathrm{~s}^{-1} \mathrm{Mpc}^{-1}\right)$. Kilborn et al. (2000) provided further evidence for this conclusion using optical imaging of J1712-64, with the goal of detecting a stellar component. To limits of $R \sim 19.2$ and $B \sim 19.8$, no overdensity of stellar sources was detected in the vicinity of J1712-64. However, at a distance of $3 \mathrm{Mpc}$, the brightest putative asymptotic giant branch (AGB) or red giant branch (RGB) stars typically found in nearby dwarf galaxies would be at $R$ magnitudes of $\sim 23-24$ well below the limit of their optical data. Any unresolved low surface brightness signature due to fainter stars would also be close to their limiting surface brightness for detection, making the null optical result somewhat ambiguous.

In an effort to acquire significantly deeper optical imaging we imaged the region around J1712-64 with the Wide Field Imager (WFI) mosaic camera at the AAT. This paper presents the new, deep images of J1712-64 obtained from these observations. The observations were processed using a new data pipeline at the Anglo-Australian Observatory, a brief synopsis of which is given in Section 2. An analysis of the images is presented in Section 3, and we finish with a brief discussion and summary of the results in Section 4.

\section{Data Reduction}

The data were obtained on the nights of August 17192001 using the WFI, mounted at the prime focus of the AAT at Coonabarabran (see Tinney 2001). The 1/2 degree $\times 1 / 2$ degree field of view of the WFI on the AAT make it an ideal instrument for searching for optical counterparts of HVCs. For the observations of HIPASS J1712-64 the seeing was sub-arcsec, but high intermittent cirrus made accurate photometric calibration difficult, and the calibration of the colour-magnitude diagrams and various associated limits alluded to later in the paper are only accurate at the $\sim 0.1$ magnitude level.

A total integration of $2700 \mathrm{~s}$ for both $g$ and $r$ bands was acquired, split as $9 \times 300 \mathrm{~s}$ exposures in each band. The field was centred at 1712 35.7, -64 3812 (J2000), corresponding to the centre of the northern component of J1712-64 (Kilborn et al. 2000). All the data were processed through a prototype AAO/WFI pipeline based on the data pipeline developed for the Isaac Newton 
Telescope Wide Field Camera (INT WFC). The pipeline provides the standard tools for removing the instrumental signature of CCD images and additionally provides means for defringing the data (if needed), object catalogue generation, and the ability to provide astrometric and photometric calibration in an automated manner (see Irwin \& Lewis 2001 for more details).

As an example of the versatility of the pipeline, the main end products in this case were a pair of stacked $g$ and $r$ band images, based on an accurate World Coordinate System (WCS) to coordinate the stacking, and the generation of a deep, detected-object colour-magnitude diagram.

\section{Image Analysis}

A false colour image of the CCD encompassing J1712-64, with the HI contours overlaid, is shown in Figure 1, using $r, 0.5(g+r)$, and $g$ for the RGB colours. Careful visual inspection of the whole mosaic image (four times the area shown in Figure 1) reveals no compelling evidence for any dwarf galaxy, resolved or otherwise, that could be associated with the HI envelope.

Morphological classification as part of the data reduction pipeline is based on automatic analysis of a discreetly sampled intensity curve-of-growth of all images. In any pairwise combination of such measures, stellar images lie on a well-defined (and constant) locus as a function of magnitude leaving only the spread (sigma) in the locus

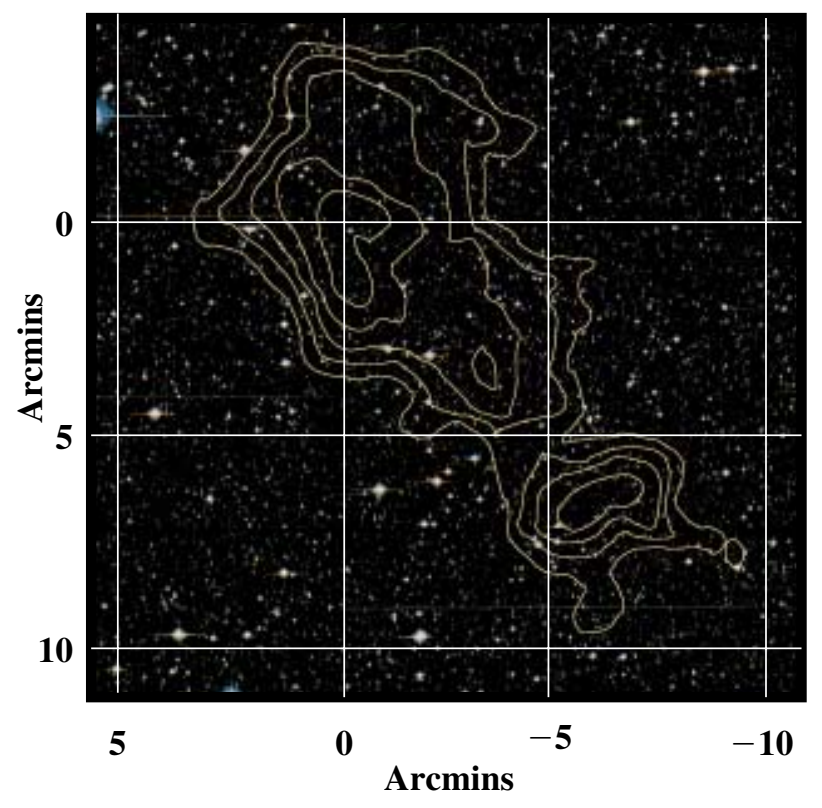

Figure 1 A false colour image of the HVC region obtained using the pipeline-processed stacked $g$ and $r$ band images to generate a colour optical image of the region. The HI contours from Kilborn et al. (2000) are overlaid. There is no obvious optical counterpart visible in either resolved stars down to $r=24, g=25$, or as a diffuse low surface brightness enhancement down to a level of $r=28$, $g=29$ magnitudes $\operatorname{arcsec}^{-2}$ respectively. The grid is relative to the centre of the CCD [17 $1235.7,-643812$ (J2000)] and the area covered in this figure is about $15 \times 15$ arcmin. to be further derived. This greatly facilitates image classification based on the sigma normalised distance from the locus. The size and 'sign' of the deviation(s) are used to assign images to one of three morphological classes: stellar, non-stellar, and noise-like.

At Galactic coordinates $l=326.6^{\circ}, b=-14.6^{\circ}$, the extinction in this direction is relatively low at $\mathrm{E}(\mathrm{B}-\mathrm{V})=$ 0.11 (Schlegel, Finkbeiner, \& Davis 1998), hence all the standard Galactic populations should be readily visible, as well as large numbers of background galaxies and any possible nearby dwarf galaxy component. Therefore, we can be more quantitative in our search by using the distribution of detected stellar images in $g, r$ to probe for any non-uniformity in image distribution. Analysis of isopleth maps, however, revealed that all are consistent with noise and therefore there is no evidence of any resolved compact stellar population in J1712-64 as would be expected for a nearby dwarf galaxy. An example is shown in Figure 2 for the range $22<r<24$ and $0.5<g-r<2.0$.

A colour-magnitude diagram for the entire mosaic region containing images classified as stellar in the $r$ band is shown in Figure 3. The expected Galactic components of the thin disk $\mathrm{K} / \mathrm{M}$ stars are clearly visible as the red plume around $g-r \approx 1.5$, while the thick disk F/G stars make up the other obvious component at $g-r \approx 0.8$. The faint blue stellar population appearing at the bottom left of the plot is most likely due to misclassified blue compact galaxies at redshifts $z \approx 0.5$. This component is much stronger in the equivalent non-stellar diagram and in both has a fairly uniform distribution over the whole field making it unlikely to be related to the HVC. Once again there

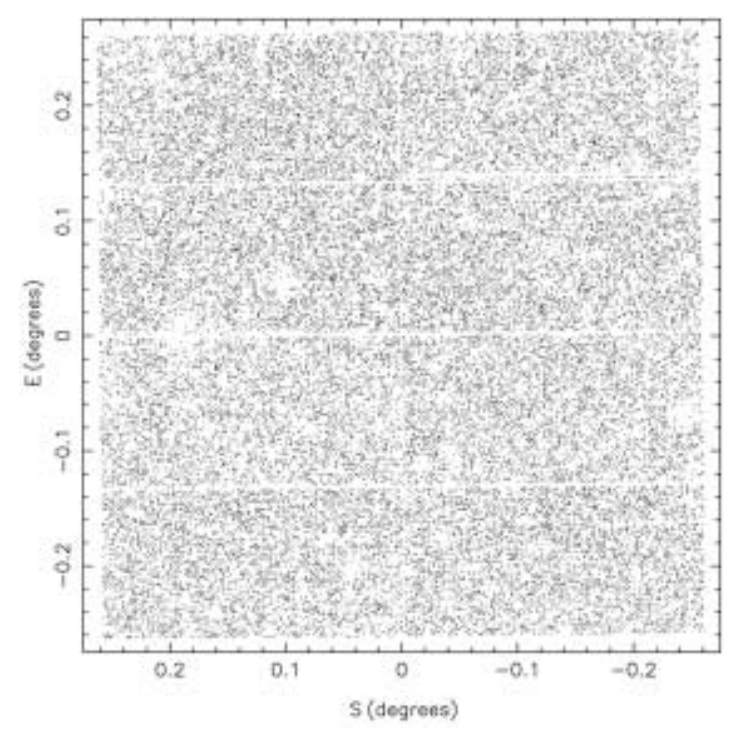

Figure 2 The spatial distribution of stars in the range $22<r<24$ and $0.5<g-r<2.0$ for the WFI image of the HVC region. Visible artifacts include the small gaps between the 8 CCDs making up the mosaic imager, regions affected by bad columns, and regions effectively masked out by the light pollution from bright foreground stars in the field. The overall distribution of stars is extremely uniform, with no evidence for any underlying resolved dwarf galaxy population. 


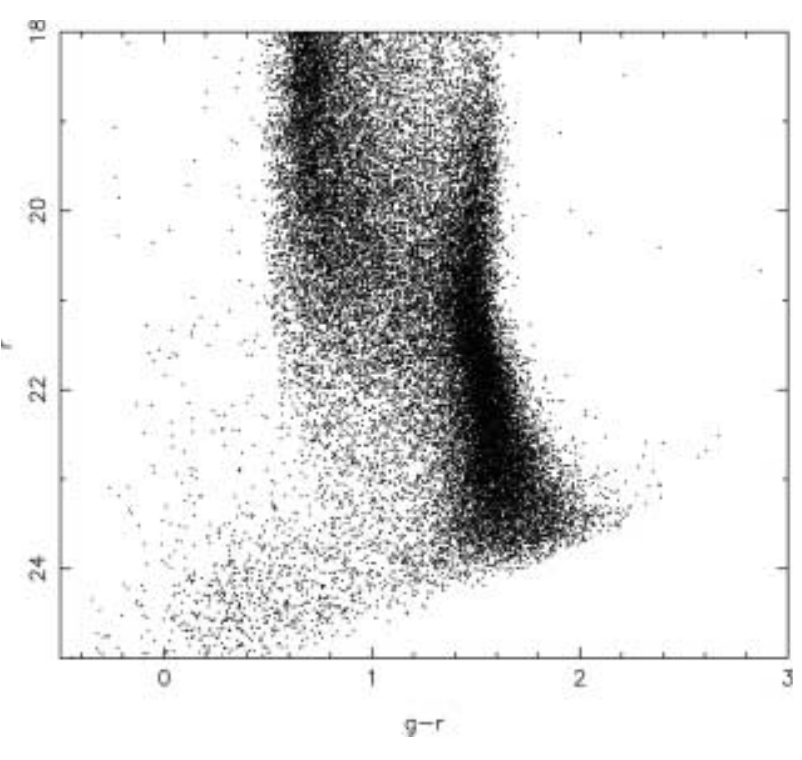

Figure 3 A $g, r$ colour-magnitude diagram for objects classified as 'stellar' in the HVC region.

is no compelling signature of any underlying dwarf galaxy component.

\section{Discussion}

This paper has presented new images of the extragalactic HI candidate HIPASS J1712-64, obtained with the WFI on the AAT. These are significantly deeper than previous images, reaching stellar magnitudes fainter than $r=24$ and $g=25$. Our search for any resolved associated stellar component was negative. Likewise, there is no evidence for any underlying unresolved diffuse components associated with the Cloud. Since the photon noise/instrumental signature surface brightness limits for detection of unresolved structure are around $r=28$ and $g=29$ magnitudes $\operatorname{arcsec}^{-2}$ respectively, we can place limits on any possible optical counterpart. In practice, the visibly patchy Galactic foreground nebulosity becomes important $\sim 1$ magnitude brighter than these limits; fortunately though, this patchiness avoids the region where the HI contours are prominent.

Typical Local Group dwarf galaxies have scale sizes of order $\sim 1 \mathrm{kpc}$ which, at a distance of $3 \mathrm{Mpc}$, would be equivalent to an angular scale of $\sim 1$ arcmin. We would expect to see the optical counterpart of such objects, if they exist, over a few scale lengths (cf. HI contours in Figure 1). Considering a 1 arcminute region and our surface brightness limits, the null detection of any diffuse optical structure leads to an absolute magnitude limit of $M_{g} \approx-7$ for this object, if it were at a distance of $3 \mathrm{Mpc}$. This is comparable to the limit derived by Kilborn et al. (2000) from photographic plates of $M_{B} \approx-9$, illustrating that we have pushed $\sim 2$ magnitudes further down the luminosity function compared to this earlier, shallower study. This limit is already at the lower end of the range for Local Group galaxies, making it unlikely than an optical component exists. Similarly, this lower limit pushes the inferred HI mass to light ratio to $>150$, at least two orders of magnitude larger than encountered in Local Group dwarf galaxies. Hence, if J1712-64 is a Local Group member, it represents a very different type of object to what is currently known.

Such conclusions make it tempting to support one obvious alternate scenario for J1712-64 — that it is a Galactic halo high-velocity cloud; a recent search of POSS plates for stellar content in a sample of 250 northern HVCs yielded no detections (Simon \& Blitz 2002). While there are a few HVCs in the HIPASS catalog (Putman et al. 2002) with Galactic standard of rest velocities comparable to that of J1712-64, should J1712-64 be a halo HVC, it would likewise necessarily be an extreme one.

It is of further interest to note that J1712-64, the earlier discovered J1616-55 (Staveley-Smith et al. 1998), and two additional clouds discovered by HIPASS (R. Koribalski et al., in preparation), all lie superimposed upon the Supergalactic Plane (SGP). An extremely deep survey of this region of the Supergalactic Plane, searching for any putative connection between the clouds, is currently underway at Parkes (R. Koribalski et al., in preparation).

The observations were processed using a new pipeline data reduction facility at the AAO. This facility is based on the reduction procedure developed for data obtained with the Wide Field Camera on the Isaac Newton Telescope on La Palma. Users of WFI at the AAT who are interested in pipeline processing their data should contact Geraint Lewis.

\section{Acknowledgments}

M. Irwin thanks the AAO for its hospitality during a visit when much of this work was finalised.

\section{References}

Blitz, L., Spergel, D. N., Teuben, P. J., Hartmann, D., \& Burton, W. B. 1999, ApJ, 514, 818

Braun, R., \& Burton, W. B. 1999, A\&A, 341, 437

Irwin, M., \& Lewis, J. 2001, NewAR, 45, 105

Kilborn, V. A., et al. 2000, AJ, 120, 1342

Klypin, A., Kravtsov, A. V., Valenzuela, O., \& Prada, F. 1999, ApJ, 522,82

Putman, M. E., et al. 2002, AJ, in press

Schlegel, D. J., Finkbeiner, D. P., \& Davis, M. 1998, ApJ, 500, 525

Sembach, K. R., Gibson, B. K., Fenner, Y., \& Putman, M. E. 2002, ApJ, in press

Simon, J. D., \& Blitz, L. 2002, ApJ, in press

Staveley-Smith, L., et al. 1998, AJ, 116, 2717

Tinney, C. G. 2001, AAO Newsletter, 96, 14 